Relations industrielles

Industrial Relations

\title{
Unions and the Public Interest: Collective Bargaining in the Government Sector, by Sandra Christensen, Vancouver, The Fraser Institute, 1980, 95 pp.
}

\section{Jean Boivin}

Volume 36, numéro 1, 1981

URI : https://id.erudit.org/iderudit/029141ar

DOI : https://doi.org/10.7202/029141ar

Aller au sommaire du numéro

Éditeur(s)

Département des relations industrielles de l'Université Laval

ISSN

0034-379X (imprimé)

1703-8138 (numérique)

Découvrir la revue

Citer ce compte rendu

Boivin, J. (1981). Compte rendu de [Unions and the Public Interest: Collective Bargaining in the Government Sector, by Sandra Christensen, Vancouver, The Fraser Institute, 1980, 95 pp.] Relations industrielles / Industrial Relations, 36(1), 275-277. https://doi.org/10.7202/029141ar

Tous droits réservés (C Département des relations industrielles de l'Université Laval, 1981
Ce document est protégé par la loi sur le droit d'auteur. L'utilisation des services d'Érudit (y compris la reproduction) est assujettie à sa politique d'utilisation que vous pouvez consulter en ligne.

https://apropos.erudit.org/fr/usagers/politique-dutilisation/ 


\section{RECENSIONS}

\section{BOOK REVIEWS}

La gestion des relations du travail au Québec. Le cadre juridique et institutionnel, par Noël Mallette (éd.), Scarborough, Collection administration, McGraw-Hill, 1980, 642 pp.

Ce n'est pas sans intérêt que nous avons parcouru cet ouvrage édité sous la direction du professeur Mallette. Il nous est possible d'affirmer qu'il y a là matière à satisfaire les intéressés aux relations du travail. Néanmoins des erreurs inacceptables de parcours transpirent.

Ainsi, il nous est apparu complètement déplacé que l'ouvrage comporte quelques travaux déjà publiés ailleurs ou ne se présentant que comme des mises à jour en surface d'écrits antérieurs. On peut aussi regretter l'importance relative qui a été accordée aux diverses présentations des auteurs. Enfin, la structure générale de l'ouvrage peut surprendre et l'on s'étonne que les questions relatives à la convention collective et à son administration (arbitrage des griefs) soient traitées avant le processus initial qui les fonde, soit la négociation collective.

Par ailleurs l'ouvrage cache des qualités indéniables. Ainsi il se présente comme un document qui prétend cerner tout à la fois les aspects juridiques et institutionnels concernant la gestion des relations du travail au Québec. C'est pourquoi y retrouve-t-on, par exemple, de bonnes présentations des structures et réalités patronales et syndicales fréquentant des essais adéquats sur le strict cadre juridique. On peut dès lors affirmer que ce recueil de textes assure une compréhension d'ensemble valable du système. Il y a certes des inégalités dans les textes; certaines résultent de la présence d'articles qui comportent des longueurs inexplicables alors que d'autres fournissent d'excellentes synthèses. Mais de façon générale la majorité des quelque 627 pages passent bien. Finalement il nous faut noter que même si cet ouvrage est avant tout le résultat d'une préoccupation pédagogique, on retrouve à l'intérieur des textes des préoccupations pratiques évidentes.

Le succès que connaîtra ce volume, qui comble un vide dans notre littérature, sera dû pour une bonne part au professeur Mallette qui a eu le courage d'entreprendre les actions nécessaires à sa réalisation. Nous ne pouvons que nous réjouir de ce nouvel apport à l'avancement des connaissances.

Rodrigue BLOUIN

Université Laval

\section{Unions and the Public Interest: Collective Bargaining in the Government Sector, by Sandra Christensen, Vancouver, The Fraser Institute, 1980, 95 pp.}

Cet ouvrage est le premier d'une série d'études entreprises par le Fraser Institute de Vancouver concernant les syndicats et l'intérêt public. Comme le titre l'indique, le livre de Sandra Christensen porte sur l'impact de la négociation collective dans le secteur public.

La démarche de l'auteur ne peut laisser le lecteur indifférent car, après avoir essayé de montrer que l'application du modèle de négociation du secteur privé au secteur des 
services gouvernementaux conduisait à des situations intolérables pour l'économie, pour la population ainsi que pour la viabilité de nos institutions démocratiques, on nous propose purement et simplement de retirer du champ de la négociation toute la question de la détermination des taux de salaires et de confier celle-ci à un tribunal spécialisé!

Même si une telle suggestion apparaît à première vue comme irréaliste et rétrograde, il faut admettre que l'argumentation développée par Sandra Christensen à l'appui de sa proposition est fondée sur des observations très pertinentes qui peuvent être difficilement démenties.

Dans un premier chapitre, l'auteur fait état de la croissance accélérée du mouvement de syndicalisation des employés du secteur public au Canada du début des années 1960 jusqu'à aujourdhui. Ainsi, en 1977, 89\% des employés non manuels et $94 \%$ des employés manuels des trois niveaux de gouvernement étaient couverts par des conventions collectives alors que la moyenne de l'ensemble des industries était respectivement de $36 \%$ et $73 \%$. Pendant la même période, le nombre d'arrêts de travail a considérablement augmenté et dans des proportions beaucoup supérieures à la stricte croissance de la maind'oeuvre employée dans ce secteur. Même si ces arrêts de travail sont de moins longue durée que dans le secteur privé, les inconvénients majeurs qu'ils causent à la population rendent les employeurs publics beaucoup plus vulnérables à accéder aux demandes syndicales ou les incitent à recourir à des législations d'urgence, d'où le sentiment général d'insatisfaction à l'égard du régime de négociation collective actuel.

Le deuxième chapitre discute des comparaisons de salaires entre les secteurs privé et public. Citant certaines études, l'auteur démontre que la rémunération des employés du secteur public a maintenant dépassé celle du secteur privé pour la presque totalité des emplois. Si l'on ajoute les avantages liés à une semaine de travail plus courte, des vacances plus longues et à une plus grande stabilité d'emploi, il faut conclure avec Sandra Christensen, que le gouvernement n'est plus seulement un bon employeur mais qu'il est devenu l'un des meilleurs. Cette situation a comme conséquence de remettre en question le principe historiquement accepté que les salaires du secteur public devraient se comparer à la moyenne de ceux payés dans le secteur privé et de faire en sorte que ce sont désormais les employés de ce secteur qui visent la parité avec ceux du secteur public.

Un troisième chapitre établit hors de tout doute ce que plusieurs savaient déjà, à savoir que la législation du travail canadienne à l'égard des employés du secteur public est la plus libérale au monde, même si à l'intérieur des onze juridictions, on retrouve des variations plus ou moins grandes.

Après avoir consacré un bref chapitre à la description du régime de négociation collective du gouvernement fédéral, l'auteur s'attaque aux problèmes posés par la transposition au secteur public du modèle de négociation du secteur privé. Ainsi, l'existence des sanctions habituelles en provenance du marché du travail permet aux syndicats d'obtenir des gains salariaux substantiels sans crainte de voir une plus ou moins grande proportion de leurs membres perdre leur emploi. D'un autre côté, parce que les coûts d'une grève pour l'employeur sont politiques plutôt qu'économiques, celui-ci sera souvent forcé de faire des concessions qui auront pour effet d'affecter l'allocation des ressources en faveur des syndiqués et au détriment de certains services dont la population sera privée.

Après avoir fait de telles critiques, l'auteur se doit quand même d'admettre que les alternatives au système actuel sont plutôt minces et c'est pourquoi, Sandra Christensen considère irréaliste de revenir à l'ancien régime de consultation où le pouvoir de décision en matière de détermination des conditions de travail demeurait unilatéralement entre les mains de l'employeur. Celle-ci rejette également le retrait global du droit de grève qui pourrait être remplacé par une forme quelconque d'arbitrage obligatoire, mais elle souligne que, s'il semble inévitable de conserver le droit de grève, on peut néanmoins prendre les moyens pour réduire la très grande vulnérabilité de l'employeur public et pour 
minimiser les inconvénients causés à la population. Parmi ces moyens qui évidemment ne feront pas l'affaire des syndicalistes, soulignons différentes actions qui pourraient être employées par l'employeur dans l'éventualité d'une grève ("contingency planning') comme des méthodes alternatives pour assurer le service, incluant le sous-contrat; la désignation d'employés essentiels; la restriction des clauses de sécurité syndicale à la simple formule Rand, de façon à pouvoir utiliser des travailleurs de l'unité de négociation qui ne supporteraient pas la grève (ce qui serait évidemment illégal au Québec actuellement); une plus grande automatisation du service. L'auteur souligne aussi la possibilité pour l'employeur de recourir à des mises à pied comme dans le secteur privé ainsi qu'à des lock outs. Enfin, elle recommande que l'employeur public n'hésite pas à se lancer dans une campagne de relations publiques pour démontrer à la population le coût exhorbitant de certaines demandes syndicales, ce qui aurait pour effet de rendre la population plus tolérante devant l'interruption de tel ou tel service et de permettre à l'employeur de mieux résister à ces demandes en supportant un plus long conflit.

Malgré cette panoplie de suggestions, Sandra Christensen admet que la plupart de ces mesures sont faibles et qu'elles contribueront sans doute très peu à réduire la vulnérabilité d'un employeur pour qui il sera habituellement plus facile d'accéder aux demandes syndicales que d'encourir les risques politiques à résister. C'est pourquoi, étant donné les caractéristiques particulières de l'emploi dans le secteur public, notamment l'absence de contraintes imposées par le marché du travail aux demandes d'augmentation de salaires des employés et croyant fermement en la validité du principe de la comparaison des salaires entre le secteur privé et le secteur public, l'auteur en arrive à proposer son système que d'aucuns qualifieront de révolutionnaire.

Selon l'auteur, si on laisse les salaires du secteur public être déterminés par le jeu de la négociation collective, cela revient tout simplement à admettre que les travailleurs membres des syndicats les plus puissants auront les meilleures conditions de travail sans égard à la situation prévalant dans le secteur privé. En faisant déterminer la rémunération par un tribunal composé de gens extérieurs aux parties syndicales et gouvernementales et jouissant d'une immunité comparable à celle des juges de la Cour suprême, on s'assure que les salaires des employés du secteur public seront alignés sur ceux du secteur privé et, selon l'auteur, on évite même que les salariés fassent les frais des politiques d'austérité des gouvernements comme ce fut le cas lors de l'imposition des contrôles anti-inflationnistes. Par ailleurs, la négociation collective, avec droit de grève, se ferait sur tous les autres éléments de la convention collective.

Le moins que l'on puisse dire de ce livre, c'est que ses conclusions ne manqueront pas de faire sursauter le lecteur. On peut peut-être lui reprocher de puiser un peu trop abondamment chez des auteurs reconnus pour la sévérité de leur jugement sur la négociation collective dans le secteur public (Wellington et Winter, par exemple), de même que déplorer certaines recommandations visant à faciliter l'utilisation de briseurs de grève. Cependant, on peut difficilement contester son parti pris en faveur de l'intérêt public; et la démonstration des vicissitudes du système de négociation collective actuel transforme graduellement la réaction négative initiale que l'on éprouve vis-à-vis sa proposition en sympathie plus ou moins consciemment avouée.

Jean BOIVIN

Université Laval

\section{A Culture in Conflict: Skilled Workers and Industrial Capitalism in Hamilton, Ontario, 1860-1914, by Bryan D. Palmer, Montreal, McGill-Queen's Uni- versity Press, 1979, $331 \mathrm{pp}$.}

Bryan Palmer is one of the ablest and most productive of the young Canadian historians currently writing the 'new' labour history pioneered by E.P. Thomson in Britain and Herbert Gutman in the United States. In brief, this approach rejects the older institutional approach to trade union history, 